\title{
A case report of a perigraft inflammatory reaction to a Viabahn stent-graft: diagnosis with MRI and treatment with steroids
}

\author{
Tetsuya Yamamoto, Kenzo Uzu* (D), Takahiro Sawada, Tomofumi Takaya and Hiroya Kawai
}

\begin{abstract}
Background: Perigraft inflammatory reactions to prosthetic graft materials in vascular surgery have been reported; however, to our knowledge, this is the first report of a perigraft inflammatory reaction to a Viabahn stent-graft used in a superficial femoral artery occlusion lesion.

Case presentation: A 76-year-old man with right leg claudication was diagnosed with a right superficial femoral artery occlusion via contrast-enhanced computed tomography. Endovascular treatment included intravascular ultrasound for passing through the true lumen. A 25-cm Viabahn stent-graft (diameter $5 \mathrm{~mm}$ ) was implanted. The patient developed pain and local swelling of the right thigh 5 days after endovascular treatment. Blood analysis revealed elevated inflammatory marker levels. Magnetic resonance imaging revealed extensive soft-tissue edema and a high perivascular T2 signal around the right superficial femoral artery. Clinical symptoms resolved within 7 days after initiating steroid therapy, which was gradually decreased and halted after 3 weeks. Follow-up magnetic resonance imaging demonstrated substantially reduced inflammation over the following months.
\end{abstract}

Conclusions: Perigraft inflammatory reaction to a Viabahn stent-graft implant can be immediately diagnosed via magnetic resonance imaging and treated with steroids to reduce the possibility of stent-graft occlusion.

Keywords: Viabahn, Perigraft inflammatory reaction, Magnetic resonance imaging, Steroid, Case report

\section{Background}

Perigraft inflammatory reactions to prosthetic graft materials in vascular surgery have been reported (Henry et al. 1994); however, to our knowledge, this is the first report of a perigraft inflammatory reaction to a Viabahn stent-graft (W.L. Gore \& Associates, Flagstaff, AZ, USA) used in a superficial femoral artery (SFA) occlusion lesion. Additionally, in the case we present here, the inflammation was diagnosed and monitored using magnetic resonance imaging (MRI) and was effectively treated with steroids.

\footnotetext{
* Correspondence: kenzouzu@gmail.com

Division of Cardiovascular Medicine, Department of Internal Medicine, Hyogo Prefectural Himeji Cardiovascular Center, 520, Saisho-Kou, Himeji, Hyogo 670-0981, Japan

Springer Open

\section{Case presentation}

A 76-year-old man presented with a peripheral occlusive disease classified as Fontaine Stage IIB. He had a history of hypertension and dyslipidemia, and was a smoker. Preoperative contrast computed-enhanced tomography (CT) revealed a right SFA occlusion (Fig. 1a). We initiated antiplatelet therapy $(100 \mathrm{mg} /$ day aspirin and $75 \mathrm{mg} /$ day clopidogrel). Angiography revealed a 16-cm chronic total occlusion of the right SFA. Endovascular treatment (EVT) was performed on the right SFA following intraarterial injection of heparin (5000 units). A 6-French (Fr) guiding sheath was inserted via the left femoral artery. Intravascular ultrasound (IVUS) was used for passing through the true lumen. Thereafter, a 4-mm semicompliant balloon was used for pre-dilatation and IVUS revealed no major dissections. A 25-cm Viabahn (diameter $5 \mathrm{~mm}$ ) was implanted and a 5-mm non-compliant 


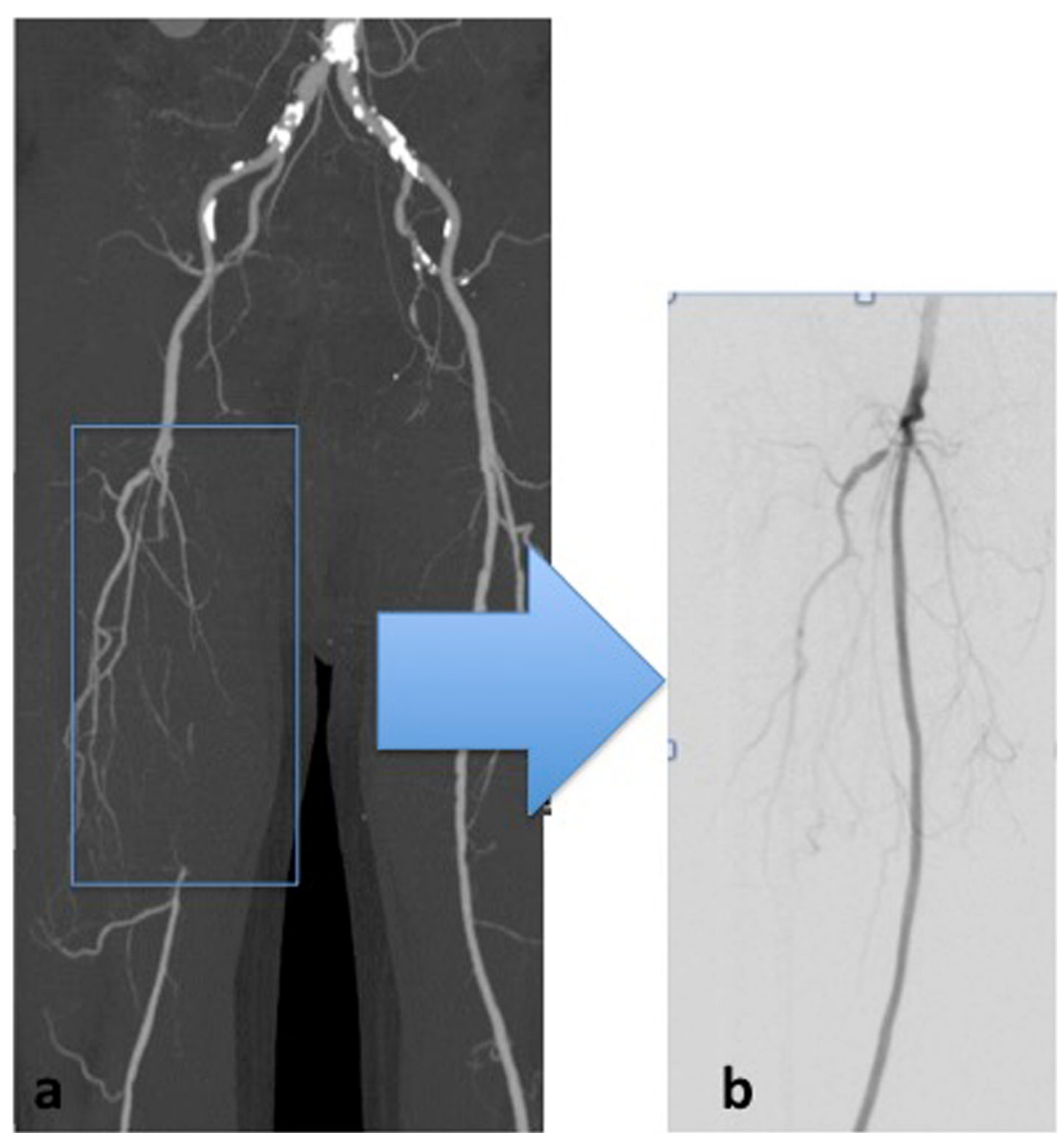

Fig. 1 Imaging of the superficial femoral artery occlusion lesion before and after endovascular therapy. a Preoperative computed tomography angiography revealing the superficial femoral artery occlusion lesion. b Post-stenting angiography revealing no dissection

balloon was used for post-dilatation. On the final angiogram, no edge dissection was visible, and good flow was confirmed (Fig. 1b). The patient's symptoms improved, and he was discharged after 4 days; however, he developed pain and local swelling of the right thigh 6 days after EVT. Venous thrombosis was excluded by ultrasound imaging. He had no fever, negative blood culture findings and negative procalcitonin levels $(0.1 \mathrm{ng} / \mathrm{ml}$; standard value is $\leq 0.5 \mathrm{ng} / \mathrm{ml}$ ). However, blood analysis revealed elevated inflammatory marker levels: white blood cell (WBC) count was 12,000 cells/ $\mu$ l (standard values are $4500-7500$ cells $/ \mu \mathrm{l}$ ) and $\mathrm{C}$-reactive protein levels were $11.83 \mathrm{mg} / \mathrm{dl}$ (standard value is $\leq 1.0 \mathrm{mg} / \mathrm{dl}$ ). MRI revealed extensive soft-tissue edema and a high perivascular T2 signal around the right SFA (Fig. 2); however, the stent-graft remained patent. This indicated possible perigraft inflammation. The clinical symptoms were severe and there was a risk of occlusion of the stent-graft. We initiated steroid therapy (prednisolone, $20 \mathrm{mg} /$ day) 2 days after the symptoms started and decreased it as soon as possible. Clinical symptoms resolved within 7 days after initiating steroid therapy, and follow-up MRI revealed regression of soft-tissue edema (Fig. 3a, b). Therefore, the prednisolone was decreased to $10 \mathrm{mg} /$ day and tapered by $5 \mathrm{mg}$ per week until it was halted. Follow-up MRI demonstrated marked resolution of inflammation over 3 months (Fig. 3c, d).

\section{Discussion and conclusions}

It is well known that local and systemic inflammation may occur after stent-graft implantation; however, to our knowledge, this is the first description of an inflammatory reaction to a Viabahn stent-graft.

There are several possible explanations for the observed inflammation. One possibility is bacterial contamination of the stent-graft during the intervention. It is difficult to 

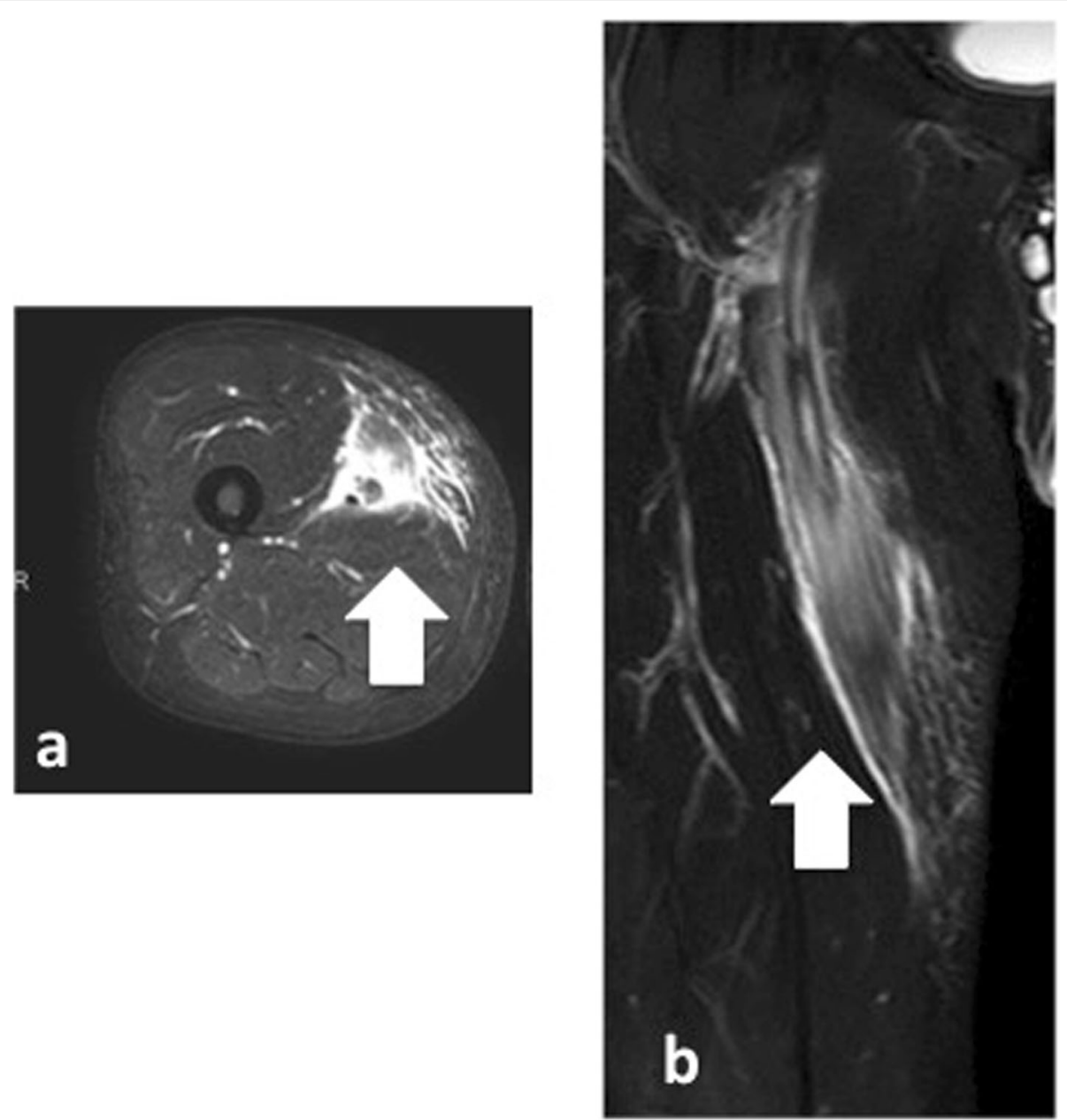

Fig. 2 T2-weighted magnetic resonance images of the patient's thigh. T2-weighted images (a, axial; $\mathbf{b}$, coronal) revealed perivascular inflammation (arrows: edema of soft tissue) surrounding the superficial femoral artery

differentiate between infection and a perigraft inflammatory reaction. However, a previous study demonstrated that patients undergoing endovascular aneurysm repair due to abdominal aortic aneurysm often develop an inflammatory response known as post-implantation syndrome (Sartipy et al. 2015). In these patients, the temperature and WBC count tended to peak on postoperative day 1 , while $C$-reactive protein and procalcitonin tended to peak on postoperative day 3. In our study, the short interval between stenting and onset of inflammation suggests a direct inflammatory reaction to the stent-graft. Additionally, repeated blood culture findings were negative, and there was no fever, no elevation of procalcitonin levels, and no evidence of a bacterial focus elsewhere in the body. Furthermore, no antibiotics were administered and clinical symptoms resolved after steroid therapy. Previously, it was reported that high-grade changes in MRI after implantation were correlated with severity of vessel wall trauma and severity of intimal dissections (Kellner et al. 1997). In the current study, neither IVUS nor postangioplasty and post-stenting angiography revealed dissection. We therefore excluded vessel wall trauma as the cause of inflammation. Another explanation is an incompatibility reaction to the polytetrafluoroethylene (PTFE) graft. Compared to polyester grafts, PTFE grafts result in a less intense inflammatory response (Sartipy et al. 2015). The Hemobahn stent-graft, which was the previous form of the Viabahn stent-graft, is made using expanded PTFE; however, inflammatory reactions to this device have been reported (Juergens et al. 2002). Additionally, unlike the Hemobahn model, the Viabahn model is heparin coated. Severe perivascular inflammation is observed more often around heparin-coated stent-grafts than around noncoated stent-grafts (Schurmann et al. 1997). Such a response may cause severe clinical symptoms or reduce patency of endovascular prostheses (Link et al. 1996a,b) and needs to be diagnosed and treated immediately. 

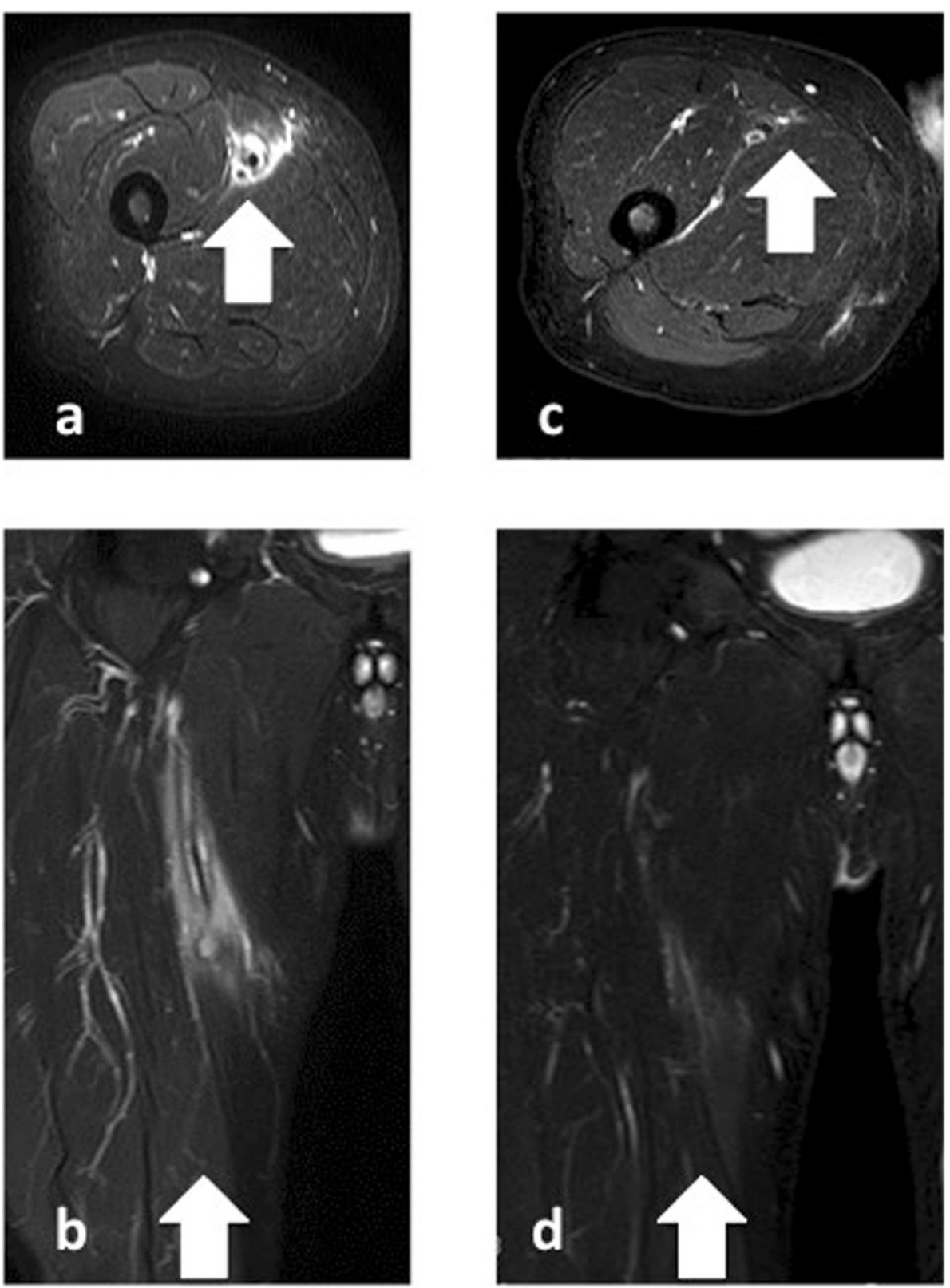

Fig. 3 Serial magnetic resonance imaging scans of the right thigh. a, b Seven days after anti-inflammatory medication was begun. c, d Three months later. Note the progressive reduction in soft-tissue edema (arrows) surrounding the right superficial femoral artery

In the present case, we evaluated the stent-graft via MRI. In a study of aortic grafts, MRI and macroscopic evaluation were compared. Macroscopic evaluation revealed a pronounced thickening of the vascular wall next to the stent-graft, and soft tissue adhesions; some cases formed a tight capsule around the Dacron-covered stent-graft due to perigraft inflammation (Schurmann et al. 1997). In the current case, soft tissue edema was also visible upon MRI. Although we could not perform histopathologic analysis, vascular wall thickening and adhesions around the Viabahn stent-graft were deemed likely upon MRI analysis.

Regarding treatment methods, it is reported that steroid treatment before implantation of a stent-graft can reduce the inflammatory response after endovascular abdominal aortic aneurysm repair, without increasing postoperative infection (Maruta et al. 2016). Steroid treatment might also be effective against a perigraft inflammatory reaction to a Viabahn stent-graft. However, such treatment increases the risk of infection (Stuck 
et al. 1989). Therefore, we initiated steroid treatment at low dosage for a short period, which immediately lowered inflammation and improved symptoms.

In conclusion, perigraft inflammatory reaction can occur in reaction to a Viabahn stent-graft implant. It can be immediately diagnosed via MRI and treated with steroids to reduce the possibility of occlusion of the stentgraft.

\section{Abbreviations}

SFA: Superficial femoral artery; MRI: Magnetic resonance imaging;

CT: Computed tomography; EVT: Endovascular treatment; IVUS: Intravascular ultrasound; PTFE: Polytetrafluoroethylene

\section{Acknowledgements}

Not applicable.

\section{Authors' contributions}

$\mathrm{KU}, \mathrm{TS}, \mathrm{T}$, and HK read and approved the manuscript.

\section{Funding}

Not applicable.

\section{Availability of data and materials}

Not applicable.

\section{Ethics approval and consent to participate}

All procedures were in accordance with the ethical standards of the institutional and with the 1964 Helsinki declaration. Informed consent was obtained from the patient in this case.

\section{Consent for publication}

Written informed consent was obtained from the patient for publication of this case report and any accompanying images. A copy of the consent form is available for review by the Editor of this journal.

\section{Competing interests}

The authors declare that they have no competing interests.

Received: 25 May 2020 Accepted: 15 July 2020

Published online: 20 September 2020

\section{References}

Henry M, Amor M, Ethevenot G, Henry I, Abdelwahab W, Leborgne E et al (1994) Initial experience with the Cragg Endopro system 1 for intraluminal treatment of peripheral vascular disease. J Endovasc Surg 1:31-43

Juergens KU, Tombach B, Reimer P, Vestring T, Heindel W (2002) Perivascular inflammatory reaction to a Hemobahn stent-graft: diagnosis with 3D MR angiography. J Endovasc Ther 9:912-916

Kellner W, Küffer G, Pfluger T, Rosa FT, Hahn K (1997) MR imaging of soft-tissue changes after percutaneous transluminal angioplasty and stent placement. Radiology 202:327-331

Link J, Feyerabend B, Grabener M, Linstedt U, Brossmann J, Thomsen $\mathrm{H}$ et al (1996a) Dacron-covered stent-grafts for the percutaneous treatment of carotid aneurysms: effectiveness and biocompatibility-experimental study in swine. Radiology 200:397-401

Link J, Müller-Hülsbeck S, Brossmann J, Steffens JC, Heller M (1996b) Perivascular inflammatory reaction after percutaneous placement of covered stents. Cardiovasc Intervent Radiol 19:345-347

Maruta K, Aoki A, Omoto T, lizuka H, Kawaura H (2016) The effect of steroid therapy on postoperative inflammatory response after endovascular abdominal aortic aneurysm repair. Ann Vasc Dis 9:168-172

Sartipy F, Lindström D, Gillgren P, Ternhag A (2015) The impact of stent graft material on the inflammatory response after EVAR. Vasc Endovasc Surg 49: $79-83$

Schurmann K, Vorwerk D, Bucker A, Neuerburg J, Klosterhalfen B, Müller G et al (1997) Perigraft inflammation due to Dacron-covered stent-grafts in sheep iliac arteries: correlation of MR imaging and histopathologic findings. Radiology 204:757-763
Stuck AE, Minder CE, Frey FJ (1989) Risk of infectious complications in patients taking glucocorticosteroids. Rev Infect Dis 11:954-963

\section{Publisher's Note}

Springer Nature remains neutral with regard to jurisdictional claims in published maps and institutional affiliations.

\section{Submit your manuscript to a SpringerOpen ${ }^{\circ}$ journal and benefit from:}

- Convenient online submission

- Rigorous peer review

- Open access: articles freely available online

High visibility within the field

- Retaining the copyright to your article

Submit your next manuscript at $\boldsymbol{\nabla}$ springeropen.com 